\title{
Food-derived bioactive peptides - a new paradigm
}

\author{
Paul J. Moughan*, Shane M. Rutherfurd, Carlos A. Montoya and Lakshmi A. Dave \\ Riddet Institute, Massey University, Private Bag 11-222, Palmerston North, New Zealand
}

\begin{abstract}
Food-derived bioactive peptides are regarded as important modulators of several physiological processes occurring both systemically and locally within the gastrointestinal tract (GIT). However, the concentrations of food-derived bioactive peptides in the GIT, and therefore attendant physiological effects, are likely to be highly variable given the wide variation in the type and amount of dietary protein consumed either during the day or on a day-to-day basis. In contrast, gut endogenous proteins (e.g. cell proteins, mucin, serum albumin and digestive enzymes) are a consistent and significant potential source of peptides for the GIT. With up to $80 \%$ of gut endogenous proteins being digested in the GIT, it is possible that a wide range of peptides is generated, but until now the significance of the gut endogenous proteins as a source of bioactive peptides has not been considered. A hypothesis is promulgated that the gut endogenous proteins may have a hidden role as a consistent and quantitatively important source of bioactive peptides in the GIT.
\end{abstract}

Key words: Bioactive peptides: Endogenous proteins: Food: Digestion

\section{Introduction}

When food proteins are digested, a myriad of variously sized peptides (di- and tripeptides, oligopeptides, to highermolecular-weight polypeptides) is released into the lumen of the digestive tract, and some may be partially resistant to further digestion. Many of these peptides that remain encrypted within the primary structure of the protein, only to be released upon digestion, have been shown to influence multiple body systems, and some share structural motifs along with endogenous peptides (peptides synthesised directly by the host organism, for example, exorphins and endorphins) known to modulate physiological function. Endogenous biologically active peptides, many produced by the action of tissue-specific proteolytic enzymes on various functional tissue proteins, are mediators of a large number of mammalian regulatory systems ${ }^{(1)}$.

Peptides, derived from food proteins, have been recovered from the intestinal digesta of animals ${ }^{(2-4)}$ and adult human subjects ${ }^{(5-8)}$. As to whether these peptides are absorbed in physiologically significant amounts remains controversial $^{(9-12)}$, and absorption may be dependent upon the size of the peptide released and other conditions. Regardless, it appears that such peptides may engage with binding sites (specific receptors) at the surface of the mucosa, leading to the induction of responses, or may have direct effects in the gut lumen itself. The reported effects of food-derived bioactive peptides are broad. They may influence the digestive, endocrine, immune, nervous and cardiovascular systems and may lead to antihypertensive, antimicrobial, lipid-lowering, immunomodulatory, mineral-binding, anti-thrombotic, opiate-like and anticariogenic effects, among others. In the gastrointestinal tract (GIT) anti-diarrhoeal effects and effects on gastrointestinal motility and gastric and pancreatic secretions, and on total ileal endogenous protein output have been reported ${ }^{(13)}$.

The generation, during digestion, of bioactive peptides from foods and their subsequent effects have been the subject of extensive review ${ }^{(12,14-18)}$.

The most studied protein source shown to give rise to bioactive peptides during digestion is milk. When milk is digested in the GIT, peptides are released that may have a range of biological activities. The two most studied groups of peptides from milk are the angiotensinconverting enzyme (ACE)-inhibiting antihypertensive peptides and the family of opioid-like peptides. These two groups are discussed here as exemplars. ACE is a multifunctional enzyme that has a key role in regulating a number of endogenous peptides. Several peptides released upon the natural digestion of milk have been shown to be ACE inhibitory, and are considered, along with several endogenous peptides, to be competitive substrates for ACE. The amino acid sequences and ACE inhibition (halfmaximal inhibitory concentration; $\mathrm{IC}_{50}$ ) of several of these bioactive peptides have been characterised ${ }^{(19)}$. Moreover, an antihypertensive effect has been demonstrated in

Abbreviations: ACE, angiotensin-converting enzyme; GIT, gastrointestinal tract. 
both animals and human subjects after oral dosage of such bioactive peptides ${ }^{(9,20)}$.

Opioid-like peptides are opioid receptor ligands with agonistic or antagonistic properties. These peptides, also appearing during the normal digestion of milk proteins, give rise to naloxone-inhibitable opioid activities ${ }^{(19)}$. The 'atypical' opioid-like peptides that are released from the hydrolysis of food and milk proteins have N-terminal sequences which are different from those of the 'typical' endogenous opioid peptides (e.g. the endorphins). A common structural feature of both the endogenous and exogenous opioid peptides is the presence of a tyrosine residue at the amino terminal end, and an aromatic residue (e.g. phenylalanine or tyrosine) in the third or fourth position. Such a structural motif is necessary for binding to the opioid receptors ${ }^{(19)}$. The opioid receptors are widely distributed throughout the mammalian body and in the nervous, endocrine, immune and digestive systems ${ }^{(14)}$. The high-resolution crystal structures of the opioid receptors and a proposed binding mode for the opioids have recently been reported ${ }^{(21)}$. Milk- and food-protein peptide derivatives with opioid agonist or antagonist activity along with opioid receptor selectivity are well characterised ${ }^{(22)}$. Several studies have demonstrated biological responses in animals and human subjects related to food-derived opioid-like peptides ${ }^{(14)}$. Effects on gastric motility, GIT secretions and GIT function in particular have been studied $^{(13,16,20,23)}$.

That milk is a rich source of bioactive materials, including bioactive peptides, may not be surprising when it is considered that milk is a biological fluid specifically designed to aid the growth and development of the neonate. Perhaps what is more surprising is that bioactive peptides arising during digestion are also commonly found in food proteins other than milk. The presence of bioactive peptides has been demonstrated in a range of diverse food protein sources including meat, eggs, cereals, bone, collagen, legumes, vegetables, marine products, yeast, seaweed and fungi. Different food proteins seem to give rise to different types of bioactive peptides during digestion and in different amounts, and some proteins may be particularly poor sources of bioactive peptides while others are particularly rich sources ${ }^{(24)}$. For an individual consuming a varied diet, the digestive tract is exposed daily to a number of bioactive peptides arising from digestion of the food proteins. It is likely, however, with vagaries in the consumption of types of dietary proteins, that such peptide production will vary among individuals and, within an individual, from day to day and potentially seasonally. The present review questions the role of dietary proteins as the sole source of bioactive peptides in the GIT, i.e. exogenous bioactive peptides, and puts forward a hypothesis that the gut endogenous proteins may have an as-yet unexplored, hidden role as a consistent source of bioactive peptides in the GIT.
Physiological significance of the food-derived bioactive peptides

The digestion of food proteins has the potential to generate high luminal concentrations of food-derived peptides; thus, although food-derived bioactive peptides may have a lower affinity for cellular receptors compared with their endogenous counterparts ${ }^{(14,21)}$, such exogenous peptides do have the potential to bring about physiologically significant effects ${ }^{(15,21,25)}$. For example, Meisel \& FitzGerald ${ }^{(14)}$ have shown that theoretically $33 \mathrm{mg}$ of $\beta$-casomorphin-7 (an opioid peptide) can be derived from the digestion of $1 \mathrm{~g}$ of $\beta$-casein, which although not a significant amount of material nutritionally, is potentially significant physiologically. Different peptides appearing in the lumen of the digestive tract may also act synergistically and potentially by interaction with the proteins themselves. Bioactive peptides derived from food proteins are numerous, often having overlapping functional activities and targets, and with some particular peptides being multifunctional. Such multiplicity of actions implies a degree of redundancy in the system.

It is an interesting thought as to whether mammals have evolved to adapt to their food source, such that exogenous food-derived peptides complement endogenous physiological regulation and control. Maybe, the very products of protein digestion in the gut lumen have a hitherto unappreciated direct role in regulating the digestive breakdown of the parent protein (co-regulation by the protein of its own digestion). More evidence is required, however, before it can be concluded that food-derived bioactive peptides are an important or perhaps even necessary component of the mammalian physiological regulatory and modulatory systems ${ }^{(14)}$, but accumulating experimental observations have led some to speculate that this may be so. In the case of gut antimicrobial activity, Pellegrini ${ }^{(25)}$ has concluded that food-derived peptides should be considered as a component of 'nutritional immunity'. Teschemacher ${ }^{(22)}$ in his classic review referred to foodderived opioid peptides as potential 'exogenous supplements to the endogenous opioidergic systems of the human organism' and further: 'a gastrointestinal role of food protein-derived opioid receptor ligands is likely'. Bos et al. ${ }^{(20)}$ conclude: 'Research conducted during the last 30 years strongly suggested that milk and other dietary protein-derived peptides could play a functional role as specific "metabolic regulators" of various physiological functions.'

\section{A quandary}

The breast-fed human infant receives a constant and consistent supply of milk proteins, and it is understandable, therefore, that during the course of human evolution, the human infant has become genetically adapted to its preweaning sole food source and milk composition has changed to meet the infant's needs. This may explain the wide 
range and functionalities of bioactive peptides arising during milk digestion. Both the composition of breast milk and the nutritional and physiological interactions between the infant and its food are the result of genetic evolution $^{(26)}$. A role for food-derived bioactive peptides in the adult human, in the context of genetic selection, however, is less clear.

During the course of human evolution, over tens of millions of years, it is considered that our free-living preagricultural human and prehuman ancestors extracted their foods from the environment in an opportunistic manner and would have had a highly variable and seasonal diet, with frequent feast and famine events ${ }^{(27-30)}$. Being hunter-gatherers, the adults probably did not consume milk or dairy products ${ }^{(29)}$. This is the type of nutritional environment to which our species became genetically adapted, and so to evolve a system of physiological regulatory activity based on specific food proteins, which implies a consistency of supply of specific dietary protein sources during evolution, is not easily conceived. Furthermore, if as has been suggested, food bioactive peptides are important for regulating gastrointestinal and other body functions in the individual, rather than just playing an incidental role, how does the body handle the considerable day-to-day and season-to-season variation in food protein sources? Diets vary greatly between individuals and for an individual often change markedly in the short term and over a lifetime. Sometimes a particular individual may go without ingesting a particular protein source or mixture of protein sources for long periods of time, without obvious ill effect. So how, in the face of such inconsistency in food protein supply, can the bioactive peptides generated in the gut lumen be important to physiological modulation?

The answer may lie in another non-food supply of protein to the gut lumen; a source referred to as gut endogenous (of body origin) protein.

\section{A new paradigm}

Every day in the adult human, approximately as much protein as that ingested with food enters the digestive lumen directly from the body ${ }^{(31)}$. These proteins are called the gut endogenous proteins. They are subjected to digestion in the digestive tract, with about $80 \%$ of the material being digested and reabsorbed. The endogenous component constitutes a consistent supply of protein to the gut lumen. Sources of endogenous materials entering the digestive lumen are saliva, gastric secretions, bile, pancreatic secretions, mucins, sloughed epithelial cells and plasma proteins (e.g. serum albumin, immunoglobulins). In addition, a significant microbial population inhabits parts of the human digestive tract, and as such bacterial cells die and are lysed, they also offer a supply of proteins to the gut lumen. Characterisation of endogenous proteins present in the lumen of the small intestine of the adult human after ingesting a casein-based meal has recently been made ${ }^{(32)}$.

A new paradigm is proposed whereby it is hypothesised that the gut endogenous proteins provide a consistent supply of protein to the digestive lumen, which upon digestion give a consistent supply of bioactive peptides that act to complement and buffer the less consistent supply of bioactive peptides from food proteins. In this way exogenous bioactive peptides may have evolved to be an important part of physiological modulatory systems. The amount of gut endogenous protein is known to be affected by dietary factors such as the amount and type of dietary fibre, the presence of antinutritional factors (e.g. tannins, lectins, trypsin inhibitors), and the amount (in general) of protein or peptides ingested. It is interesting to note that peptides released from food proteins, and the dietary concentrations of these peptides, have been shown to quantitatively increase the amounts of endogenous (body) proteins present in the digestive lumen ${ }^{(33-36)}$. This would infer that the food-derived peptides may modulate the production of peptides from the gut endogenous proteins, and that the two sources of bioactive peptides (dietary and gut endogenous) may be interactive rather than separate systems.

\section{Evidence for bioactive peptides derived from gut endogenous proteins}

Our group has undertaken in silico studies of proteins representative of the gut endogenous proteins ${ }^{(37)}$. Based on their known amino acid sequences and digestive enzyme cleavage sites, it is clear that many of these proteins are able to give rise, during digestion, to many and a wide range of peptides.

The most notable observation of this in silico study was that the number and composition of bioactive peptide fragments deemed to be released from the amino acid sequences of both gut endogenous proteins and dietary sources were remarkably similar. By way of example, when bovine $\beta$-casein (an extensively studied food protein source of bioactive peptides) was compared to human serum albumin (an endogenous protein present in the human GIT), using the in silico approach and based on the specificity of pepsin, trypsin and chymotrypsin, it was predicted that similar numbers of bioactive peptides would potentially be released from the total 231 bioactive peptide sequences present in one molecule of $\beta$-casein and the total 339 bioactive peptide sequences per molecule of human serum albumin, after digestion in the GIT.

In an attempt to put the potential of gut endogenous proteins as a source of bioactive peptides into perspective, the quantity of bioactive peptides derived from the gastrointestinal digestion of gut endogenous proteins and from a model diet was predicted using the in silico approach. The model diet comprised of milk, wheat, soya, egg and meat and the bioactive peptides present, post-digestion, were 
predicted for the major proteins present in these foods. Similarly, bioactive peptides present in the gut, postdigestion, were also predicted based on the reported composition of gut endogenous proteins and the reported digestibility of these proteins ${ }^{(32)}$. It was estimated that a total of $2563 \mathrm{mg}$ of bioactive peptides per $\mathrm{d}$ would be generated from dietary proteins, while $5396 \mathrm{mg}$ of bioactive peptides per $\mathrm{d}$ would be generated from gut endogenous proteins. This preliminary analysis indicates that gut endogenous proteins may be at least as important as the dietary proteins as a source of bioactive peptides in the GIT, particularly given that the gut endogenous proteins will almost certainly be present more consistently than proteins of dietary origin. Moreover, when viewed in the light of the proposed peptidergic system of regulation of various physiological processes ${ }^{(1)}$, our findings could explain a possible secondary role for the diverse and continuously present gut endogenous proteins. The observed abundance of bioactive peptide fragments in the amino acid sequences of gut endogenous proteins may infer a buffering ability, in the event of a variable supply of bioactive peptides arising from the dietary proteins.

Clearly the in silico analysis only speaks to the potential for the generation of bioactive peptides from gut endogenous proteins, and takes no account of the effects of secondary and tertiary protein structures on the rate of digestion and subsequent peptide release. Nor does it take into account the effect of bacterial proteases present in the GIT lumen. It does appear, however, based on the many peptides identified that are homologous to food-derived bioactive peptides, that the gut endogenous proteins are a substantial potential source of bioactive peptides in the gut lumen. This source of protein for the digestive tract has the potential to supply a continuous stream of bioactive peptides.

The conduct of in vivo studies is needed to demonstrate the release during digestion of such bioactive peptides. In this respect it is interesting to note that, in an in vitro study, a peptic digest of bovine serum albumin gave rise to a material demonstrating opioid activity ${ }^{(38)}$. This compound was subsequently identified as the opioid agonist named serorphin. It has also been shown in ruminants that an abomasal infusion of saliva (an endogenous gut protein) leads to an increase in the frequency and duration of reticular contractions ${ }^{(23)}$ thought to be opioid mediated.

\section{Conclusions}

It appears that exogenous food-derived bioactive peptides may have numerous physiological modulatory and regulatory activities and these effects may be complemented by gut endogenous protein-derived bioactive peptides. If the latter hypothesis holds up to further empirical investigation, it will be illuminating to examine which peptides are released from the gut endogenous proteins in vivo and in what amounts. This may better highlight which gut-derived bioactive peptides are most important physiologically. Much work needs to be undertaken on the isolation, characterisation, and definition of mechanisms. Once structures and mechanisms of action have been elucidated, it will be useful to see just what foods under what conditions augment the effects of the bioactive peptides originating directly from the gut endogenous proteins.

\section{Acknowledgements}

This research received no specific grant from any funding agency in the public, commercial or not-for-profit sectors.

The original idea and hypothesis are of P. J. M. and L. A. D. undertook the data analysis under the supervision of S. M. R. and C. A. M. All authors contributed to the writing. There are no conflicts of interest.

\section{References}

1. Karelin AA, Blishchenko EY \& Ivanov VT (1998) A novel system of peptidergic regulation. FEBS Lett 428, 7-12.

2. Yvon M \& Pelissier JP (1987) Characterization and kinetics of evacuation of peptides resulting from casein hydrolysis in the stomach of the calf. J Agric Food Chem 35, 148-156.

3. Scanff P, Yvon M, Thirouin S, et al. (1992) Characterization and kinetics of gastric emptying of peptides derived from milk proteins in the preruminant calf. $J$ Dairy Res 59, 437-447.

4. Meisel H (1986) Chemical characterization and opioid activity of an exorphin isolated from in vivo digests of casein. FEBS Lett 196, 223-227.

5. Svedberg J, de Haas J, Leimenstoll G, et al. (1985) Demonstration of $\beta$-casomorphin immunoreactive materials in in vitro digests of bovine milk and in small intestine contents after bovine milk ingestion in adult humans. Peptides $\mathbf{6}$, $825-830$

6. Mahe S, Benamouzig R, Gaudichon C, et al. (1995) Nitrogen movements in the upper jejunum lumen in humans fed low amounts of casein or $\beta$-lactoglobulin. Gastroenterol Clin Biol 19, 20-26.

7. Chabance B, Marteau P, Rambaud JC, et al. (1998) Casein peptide release and passage to the blood in humans during digestion of milk or yogurt. Biochimie 80, 155-165.

8. Boutrou R, Gaudichon C, Dupont D, et al. (2013) Sequential release of milk protein-derived bioactive peptides in the jejunum in healthy humans. Am J Clin Nutr 97, 1314-1323.

9. Martínez-Maqueda D, Miralles B, Recio I, et al. (2012) Antihypertensive peptides from food proteins: a review. Food Funct 3, 350-361.

10. Jahan-Mihan A, Luhovyy BL, Khoury DE, et al. (2011) Dietary proteins as determinants of metabolic and physiologic functions of the gastrointestinal tract. Nutrients 3, 574-603.

11. Walther B \& Sieber R (2011) Bioactive proteins and peptides in foods. Int J Vitam Nutr Res 81, 181-192.

12. Möller NP, Scholz-Ahrens KE, Roos N, et al. (2008) Bioactive peptides and proteins from foods: indication for health effects. Eur J Nutr 47, 171-182.

13. Moughan PJ, Fuller MF, Han KS, et al. (2007) Food-derived bioactive peptides influence gut function. Int J Sport Nutr Exerc Metab 17, Suppl., S5-S22.

14. Meisel H \& FitzGerald RJ (2000) Opioid peptides encrypted in intact milk protein sequences. BrJ Nutr 84, S27-S31. 
15. Meisel H (2005) Biochemical properties of peptides encrypted in bovine milk proteins. Curr Med Chem 12, 1905-1919.

16. Rutherfurd-Markwick KJ \& Moughan PJ (2005) Bioactive peptides derived from food. J AOAC Int $\mathbf{8 8}$, 955-966.

17. Shahidi F \& Zhong Y (2008) Bioactive peptides. J AOAC Int 91, 914-931.

18. Korhonen H (2009) Milk-derived bioactive peptides: from science to applications. J Funct Foods 1, 177-187.

19. Meisel H (1997) Biochemical properties of regulatory peptides derived from milk proteins. Biopolymers 43, 119-128.

20. Bos C, Gaudichon C \& Tome D (2000) Nutritional and physiological criteria in the assessment of milk protein quality for humans. J Am Coll Nutr 19, 191S-205S.

21. Filizola M \& Devi LA (2012) Structural biology: how opioid drugs bind to receptors. Nature 485, 314-317.

22. Teschemacher H (2003) Opioid receptor ligands derived from food proteins. Curr Pharm Des 9, 1331-1344.

23. Froetschel MA (1996) Bioactive peptides in digesta that regulate gastrointestinal function and intake. J Anim Sci $\mathbf{7 4}$, 2500-2508.

24. Korhonen H \& Pihlanto A (2003) Food-derived bioactive peptides - opportunities for designing future foods. Curr Pharm Des 9, 1297-1308.

25. Pellegrini A (2003) Antimicrobial peptides from food proteins. Curr Pharm Des 9, 1225-1238.

26. Goldman AS (2000) Modulation of the gastrointestinal tract of infants by human milk. Interfaces and interactions. An evolutionary perspective. J Nutr 130, 426S-431S.

27. Milton K (2000) Back to basics: why foods of wild primates have relevance for modern human health. Nutrition $\mathbf{1 6}$, 480-483.

28. Garn SM \& Leonard WR (1989) What did our ancestors eat? Nutr Rev 47, 337-345.
29. Eaton SB (1990) What did our late paleolithic (preagricultural) ancestors eat? (I). Nutr Rev 48, 227-229.

30. Eaton SB \& Eaton SB III (2000) Paleolithic vs. modern diets selected pathophysiological implications. Eur J Nutr 39, $67-70$.

31. Moughan PJ \& Rutherfurd SM (2012) Gut luminal endogenous protein: implications for the determination of ileal amino acid digestibility in humans. BrJ Nutr 108, S258-S263.

32. Miner-Williams W, Deglaire A, Benamouzig R, et al. (2012) Endogenous proteins in terminal ileal digesta of adult subjects fed a casein-based diet. Am J Clin Nutr 96, 508-515.

33. Hodgkinson SM \& Moughan PJ (2007) An effect of dietary protein content on endogenous ileal lysine flow in the growing rat. J Sci Food Agric 87, 233-238.

34. Butts CA, Moughan PJ, Smith WC, et al. (1993) The effect of food dry matter intake on endogenous ileal amino acid excretion determined under peptide alimentation in the $50 \mathrm{~kg}$ liveweight pig. J Sci Food Agric 62, 235-243.

35. Skilton GA, Moughan PJ \& Smith WC (1988) Determination of endogenous amino acid flow at the terminal ileum of the rat. J Sci Food Agric 44, 227-235.

36. Hodgkinson SM, Moughan PJ, Reynolds GW, et al. (2000) The effect of dietary peptide concentration on endogenous ileal amino acid loss in the growing pig. Br J Nutr 83, 421-430.

37. Acharya L, Montoya CA, Rutherfurd SM, et al. (2012) Endogenous proteins: are they a source of bioactive peptides? In Proceedings of the Food Structures, Digestion and Health International Conference. Palmerston North: Riddet Institute, Palmerston North, New Zealand, 7-9 March 2012, 64 .

38. Zioudrou C, Streaty RA \& Klee WA (1979) Opioid peptides derived from food proteins. The exorphins. $J$ Biol Chem 254, 2446-2449. 